\title{
ON FACTORIZATION OF MEROMORPHIC FUNCTIONS WITH FINITELY MANY POLES
}

\author{
ANAND P. SINGH AND K. S. CHARAK*
}

\begin{abstract}
In this paper we give a criterion for determining the pseudo-primeness of meromorphic functions with finitely many poles. The criterion depends on the dispersion of the zeros of the function, more spcifically on the Picard sets for entire functions. Also, we give some applications of this criterion.
\end{abstract}

\section{Introduction}

A meromorphic function $F(z)=f(g(z))$ is said to have $f$ and $g$ as left and right factors respectively provided $f$ is meromorphic and $g$ is entire ( $g$ may be meromorphic, when $f$ is rational). $F$ is said to be pseudo-prime (right pesudo-prime, right-prime), if every factorization of the above form implies either $f$ is rational or $g$ is a polynomial ( $g$ is polynomial whenever $f$ is transcendental, $g$ is linear whenever $f$ is transcendental).

In order to prove that a given function $F$ is pseudo-prime, the following two powerful theorems are frequently used.

Theorem A. (Goldstein). Let $F$ be an entire function of finite order with $\delta(a, F)=1$, for some complex number $a$. Then $F$ is pseudo-prime.

Theorem B. (Steinmetz). Let $n$ be a positive integer and

$$
w^{(n)}(z)+A_{n}(z) w^{(n-1)}(z)+\cdots+A_{1}(z) w(z)+A_{0}(z)=0 .
$$

be a linear differential equation in $w$ with all the coefficients $A_{i}(z),(i=0,1, \ldots)$ being rational functions. Then any meromorphic solution $f$ of (1) must be pseudo-prime.

As is evident, Theorem A is applicable only if $F$ is a finite ordered entire function, whereas in Theorem B the meromorphic function must satisfy (1). Also by [9, p.73], the meromorphic function satisfying (1) must be of finite order. Thus, as far as we are aware, there is no criteria for determining the pseudo-primeness of functions of infinite order. We give in Theorem 1 below, a criterion which is completely different from that given either by Goldstein or by Steinmetz, and which is applicable to functions of infinite order as well.

Received April 4, 1996.

1991 Mathematics Subject Classification. Primary 30D05.

* The work of the second author is supported by C. S. I. R., Govt. of India, through S. R. F., Grant No. 9/100(64)/95-EMR-I. 
Apart from the properties mentioned in Theorem A and Theorem B, we shall show that the pseudo-primeness of certain meromorphic functions also depends on the location and dispersion of their zeros.

Before embarking on the statements of our results, we shall recall the concept of Picard sets for entire functions.

Definition A. A set $S$ in the complex plane $\mathbb{C}$ is called Picard set for transcendental entire functions if every transcendental entire function assumes every complex value with at most one exception, infinitely often in $\mathbb{C} \backslash S$. Various Picard sets are known to exist, see for instance [1], [2], [3], [7], [8] and their references.

Throughout this paper, by a really meromorphic function we shall mean a meromorphic function in $\mathbb{C}$ having at least one pole.

\section{Statements of Results}

Theorem 1. Let $S$ be a Picard set for entire functions. Let $f$ be a transcendental really meromorphic function having finitely many poles, and with infinitely many zeros all lying in $S$. Then $f$ is right pseudo-prime.

Remark 1 . Thus for instance all transcendental really meromorphic functions having finitely many poles and infinitely many zeros all lying in $E$, where $E$ is defined by various conditions in Theorems 1-4, Theorems 10-11 of [7], are right pseudo-prime. Similarily, transcendental meromorphic functions with finitely many zeros and infinitely many poles all lying in $E$ are also right pseudo-prime.

As an application of Theorem 1, we shall prove.

Theorem 2. Let $f$ be a transcendental really meromorphic function with finitely many poles and infinitey many zeros, $\left\{z_{n}\right\}$ all of which satisfy

$$
\left|z_{n+1}\right| \geq k\left|z_{n}\right|
$$

for some $k>1, n=1,2, \ldots$; Then $f$ is right-prime.

Remark 2. Note that

$$
f(z)=\frac{\sin \left(z e^{z}+1\right)}{z e^{z}},=\frac{\sin (z+1)}{z} \circ z e^{z},
$$

shows that the condition (2) is essential.

Remark 3. Let

$$
F(z)=\frac{f(z) \exp (f(z))}{P(z) \exp (P(z)(1+\exp (P(z)))}
$$

where $f$ is an entire function with zeros $\left\{z_{n}\right\}$ as in Theorem 2 and $P(z)$ be a non-constant poynomial with zeros distinct from $z_{n}$ 's. Then the numerator and denominator of $F$ in (3) are both composite, and yet, the function $F$ itself is right-prime. 
Remark 4. Theorem 2 is not true for entire functions. For let $P(z)$ be the canonical product formed by the zeros $\left\{z_{n}\right\}$ of Theorem 2 . Then

$$
f(z)=P(z) e^{P(z)}=z e^{z} \circ P(z),
$$

shows that $f$ is not pseudo-prime.

For transcendental meromorphic functions whose all zeros and poles lie in a Picard set for entire functions, we shall prove the following.

Theorem 3. Let $f$ be a transcendental really meromorphic function with at least one zero. Further suppose that all the zeros and poles of $f$ lie in a Picard set $S$ for entire functions. Then $f$ has to be pseudo-prime.

Finally, we shall prove a related result which deals with the dispersion of the zeros of $F$ and its right factor.

Theorem 4. Let $\left\{a_{n}\right\}$ be a complex sequence and $\left\{\rho_{n}\right\}$ be a positive sequence satisfying, for all $n$,

$$
\left|\frac{a_{n+1}}{a_{n}}\right|>q>1
$$

and

$$
\log \frac{1}{\rho_{n}}>\frac{q^{1 / 4}+1}{q^{1 / 4}-1} \cdot \frac{8}{\log q}\left(\log \left|a_{n}\right|\right)^{2}
$$

and let $N \geq 2$ be an integer. Let $F$ be a transcendental really meromorphic function with finite number of poles and $\left\{a_{n}\right\}$ as its zeros. Then $F$ cannot have $f^{N} f^{\prime}$ as its right factor for every transcendental entire function $f$.

\section{Proofs of Theorems}

Proof of Theorem 1. Let $f=g o h$, where $g$ is transcendental meromorphic and $h$ is transcendental entire. By hypothesis of Theorem 1, it easily follows that $g$ has at least one zero. We assert that $g$ has exactly one zero $w_{0}$, say. For, if $g$ has two zeros $w_{1}$ and $w_{2}\left(w_{1} \neq w_{2}\right)$, then at least one of $w_{i}(1=1,2$.$) say w_{1}$ will be such that $h(z)=w_{1}$ will have infinitely many solutions outside $S$. But then these solutions are zeros of $f$, which is a contradiction to the hypothesis that all the zeros of $f$ lie in $S$.

Next, if $g$ has a pole $\zeta_{0}$, say, then $\zeta_{0} \neq w_{0}$ and so there exist infinitely many complex numbers $\xi_{n},(n=1,2 \ldots)$ obviously outside $S$ such that $h\left(\xi_{n}\right)=\zeta_{0}$. But then $\left\{\xi_{n}\right\}$ are poles of $f$. This contradiction proves that $f$ is pseudo-prime.

Further, if $f=g o h$, where $g$ is rational, then clearly $g$ has at least one zero $\eta_{0}$ and at least one pole $\zeta_{0}$, say. Now $\left\{h^{-1}\left(\eta_{0}\right)\right\}$ is contained in $S$ and consequently there are infinitely many complex numbers in $\left\{h^{-1}\left(\zeta_{0}\right)\right\}$ which lie outside $S$. Since $\left\{h^{-1}\left(\zeta_{0}\right)\right\}$ is an infinite set of poles of $f$, it follows that $f$ has infinitely many poles. This contradiction proves Theorem 1. 
Proof of Theorem 2. Consider a positive sequence $\left\{d_{n}\right\}$ such that

$$
\log \frac{1}{d_{n}} \geq \frac{q\left(\log \left|z_{n}\right|\right)^{2}}{\log k}, \quad n>n_{0}
$$

for some $q>1 / 2$. Let $S=U_{n=1}^{\infty} E_{n}$, where $E_{n}=\left\{z:\left|z-z_{n}\right|<d_{n}\right\}$. Then it is shown that $S$ is a Picard set for entire functions [1]. Also clearly $z_{n} \in S$ for all $n$. Thus in view of Theorem 1 , it follows that $f$ is right pseudo-prime.

If $f=g o h$, where $g$ is a transendental meromorphic function and $h$ is a polynomial of degree $d \geq 2$, then following the arguments of $L \mathrm{i}$ [6], we easily deduce that $g$ has infinitely many zeros $a_{n},(n=1,2 \ldots)$ say. For large $a_{n}, h(z)=a_{n}$ has exactly $d$ solutions $b_{1}^{(n)}, b_{2}^{(n)}, \ldots, b_{d}^{(n)}$, say, which satisfy

$$
f\left(b_{i}^{(n)}\right)=0, \quad(i=1,2, \ldots, d,)
$$

and are asymptotically equally distributed on a circle $\Gamma_{n}:|z|=A\left|a_{n}\right|^{1 / d}$, where $A$ is some constant.

Let

$$
F_{n}=\left\{z: k^{-1 / 2}\left|z_{n}\right|<|z|<k^{1 / 2}\left|z_{n}\right|\right\}, \quad(n=1,2, \ldots,)
$$

Then clearly $z_{n} \in F_{n}$ and $z_{i} \notin F_{n}$ for $i \neq n$. Thus $F_{n}$ contains only one zero of $f$. Also by (4), there exists $z_{i_{0}}$ such that $b_{1}^{(n)}=z_{i_{0}} \in F_{i_{0}}$ and so for $1 \leq i \leq d, b_{i}^{(n)} \in F_{i_{0}}$, since $b_{i}^{(n)},(i=1,2, \ldots, d)$ lie asymptotically on the circle $\Gamma_{n}$. Thus $F_{i_{0}}$ contains $d \geq 2$ zeros of $f$. This contradiction proves the theorem.

Proof of Theorem 3. Let $f=$ goh, where $g$ is transcendental meromorphic function. Since $f$ is really meromorphic, $g$ has at least one pole $b$, say. Also $g$ has a zero $a$, say. And $h-a$ and $h-b$ have all their zeros in $S$. Thus, if $h$ were a transcendental entire function, then $S$ would not be a Picard set for entire functions, contradicting the assumption. Hence $h$ has to be a polynomial.

For the proof of the next theorem, we shall need the following result of Langley [5].

Lemma. Suppose that the complex sequence $\left\{a_{n}\right\}$ and the positive sequence $\left\{\rho_{n}\right\}$ satisfy, for all $n$,

$$
\left|\frac{a_{n+1}}{a_{n}}\right|>q>1
$$

and

$$
\log \frac{1}{\rho_{n}}>\frac{q^{1 / 4}+1}{q^{1 / 4}-1} \cdot \frac{8}{\log q} \quad\left(\log \left|a_{n}\right|\right)^{2}
$$

Then, if $f$ is a transcendental entire function, and $N$ is an integer not less than 2 , the equation $f^{N}(z) f^{\prime}(z)=b$ must have infinitely many solutions outside the union of the disks $B\left(a_{n}, \rho_{n}\right)$, for any $b \neq 0$.

Proof of Theorem 4. Let $S=U_{n=1}^{\infty} B\left(a_{n}, \rho_{n}\right)$. Suppose $F=g o\left(f^{N} f^{\prime}\right)$, where $f$ is transcendental entire function and $g$ is meromorphic. By hypothesis, $g$ has at least one 
zero. We assert that $w=o$ is the only zero of $g$. For, if $c \neq o$ is another zero of $g$, then by Lemma, the equation $f^{N}(z) f^{\prime}(z)=c$ has infinity of solutions, $\{\zeta n\}$ say, outside $S$ and so $F\left(\zeta_{n}\right)=o,(n=1,2, \ldots$,$) , which contradicts the fact that all zeros of F$ lie in $S$.

Next, if $\alpha$ is a pole of $g$, then $\alpha \neq o$ and so $f^{N}(z) f^{\prime}(z)=\alpha$ will have infinity of solutions outside $S$, thereby showing that $F$ has infinitely many poles. This contradiction proves the theorem.

\section{References}

[1] J. Anderson and J. Clunie, "Picard set of entire and meromorphic functions," Ann. Acad. Sci. Fenn. Ser. A, 15(1980), 27-43.

[2] I. N. Baker and L. S. O. Liverpool, "Picard sets of entire functions," Math. Z., 126(1972), 230-238.

[3] I. N. Baker and L. S. O. Liverpool, "Further results on Picard sets of entire functions," Proc. London Math. Soc.(3), 26(1973), 82-98.

[4] C. T. Chuang and C. C. Yang, "Fix points and factorization of meromorphic functions," World Scientific, Pub. Co. Singapore, 1990.

[5] J. K. Langley, "Analogues of Picard sets for entire functions and their derivatives," Contemporary Math., Vol. 25(1983), 75-87.

[6] B. Q. Li, "Unique factorizability of the p-th power of entire functions," J. Math. Anal., Appl. 154(1991), 435-445.

[7] Sakari Toppila, "Picard sets for meromorphic functions with a deficient value," Ann. Acad. Sci. Fenn. Ser. AI Math., 5(1980), 263-300.

[8] Sakari Toppila, "Picard sets for meromorphic functions," Ann. Acad. Sci. Fenn. Ser. AI, 417(1967), 1-24.

[9] H. Wittich, "Neuere untersuchungen uber eindeutige analytische funktionen," Springer Verlag, Berlin, 1968.

Department of Mathematics, University of Jammu, Jammu-180 004, India. 